Article

\title{
Assessment of Multi-Mycotoxin Exposure in Southern Italy by Urinary Multi-Biomarker Determination
}

\section{Michele Solfrizzo *, Lucia Gambacorta and Angelo Visconti}

Institute of Sciences of Food Production (ISPA), National Research Council (CNR), Bari 70126, Italy; E-Mails: lucia.gambacorta@ispa.cnr.it (L.G.); angelo.visconti@ispa.cnr.it (A.V.)

* Author to whom correspondence should be addressed; E-Mail: michele.solfrizzo@ispa.cnr.it; Tel.: +39-080-592-9367; Fax: +39-080-592-9374.

Received: 13 December 2013; in revised form: 13 January 2014 / Accepted: 21 January 2014 / Published: 28 January 2014

\begin{abstract}
Human exposure assessment to deoxynivalenol (DON), aflatoxin B1 (AFB1), fumonisin B1 (FB1), zearalenone (ZEA) and ochratoxin A (OTA) can be performed by measuring their urinary biomarkers. Suitable biomarkers of exposure for these mycotoxins are $\mathrm{DON}+$ de-epoxydeoxynivalenol (DOM-1), aflatoxin M1 (AFM1), FB 1 , ZEA + $\alpha$-zearalenol $(\alpha-Z O L)+\beta$-zearalenol $(\beta$-ZOL) and OTA, respectively. An UPLC-MS/MS multi-biomarker method was used to detect and measure incidence and levels of these biomarkers in urine samples of 52 volunteers resident in Apulia region in Southern Italy. The presence of ZEA + ZOLs, OTA, DON, FB1 and AFM1 were detected in 100\%, 100\%, 96\%, 56\% and $6 \%$, of samples, respectively. All samples contained biomarkers of two or more mycotoxins. The mean concentrations of biomarkers ranged from $0.055 \mathrm{ng} / \mathrm{mL}$ (FB1) to $11.89 \mathrm{ng} / \mathrm{mL}$ (DON). Urinary biomarker concentrations were used to estimate human exposure to multiple mycotoxin. For OTA and DON, $94 \%$ and $40 \%$ of volunteers, respectively exceeded the tolerable daily intake (TDI) for these mycotoxins. The estimated human exposure to FB1 and ZEA was largely below the TDI for these mycotoxins for all volunteers.
\end{abstract}

Keywords: mycotoxins; biomarker; urine; UPLC-MS/MS; immunoaffinity cleanup; exposure

\section{Introduction}

Aflatoxins, deoxynivalenol (DON), zearalenone (ZEA), fumonisins and ochratoxin A (OTA) are recognized as the principal mycotoxins occurring in agricultural products and their levels in food 
commodities are constantly inspected worldwide. Humans can be daily exposed to mixtures of these mycotoxins through consumption of foods contaminated with several mycotoxins or consumption of different foods contaminated by a single mycotoxin. Data on the co-occurrence of the principal mycotoxins in foods and beverages are increasing due to the availability and use of modern and sensitive LC-MS/MS methodologies suitable for simultaneous determination of mycotoxins and other fungal metabolites $[1,2]$. In a recent survey on 265 samples of cereal-based products commercialized in Spain, Italy, Marocco and Tunisia, 14\% of the analyzed samples were contaminated with at least two mycotoxins and $18 \%$ of the analyzed samples were contaminated by more than two mycotoxins simultaneously [3]. The co-occurrence of DON, ZEA and nivalenol (NIV) in winter wheat produced in Sweden has been recently reported [4]. The presence of mixtures of aflatoxin B1 (AFB 1$)$, ZEA and OTA was reported in samples of breakfast cereals commercialized in Spain [5]. The majority of food commodities consumed in Cameroon were found contaminated with mixtures of mycotoxins, $21 \%$ contained DON, ZEA and fumonisin B1 (FB1), 11\% DON, AFB1, FB1 and ZEA [6]. Recently, the co-occurrence of DON, FB1, fumonisin B2 (FB2), fumonisin B3 (FB3) and ZEA in good and moldy maize and DON, FB1, FB2, ZEA and OTA in samples of maize based foods was reported in the former Transkei region of South Africa [7,8].

Exposure to mycotoxins can also originate from the ingestion of their masked forms (mycotoxins covalently or non-covalently bound to matrix component) that are digested in the gastrointestinal tract and released into the parent mycotoxins that become bioavailable. The occurrence of masked DON and ZEA, either acetylated, conjugated with glucose or sulfate, has been reported in various cereals and food samples [9]. The occurrence of masked fumonisins in processed food has also been reported but the nature of the masking mechanism has not been fully clarified [10]. The formation of $\beta$-glucosides of (4R)- and (4S)-5-hydroxy-OTA in germinating cereals and vegetables spiked with OTA has been demonstrated but the occurrence of these compounds in naturally contaminated food has not been reported [11]. The degree of human bioavailability of mycotoxins derived from their masked forms is not known and could vary between individuals depending of the intestinal microbiota composition.

The assessment of human exposure to mycotoxins is usually performed by means of chemical analysis of foods and beverages and results are correlated with the mean intake of analyzed foods/beverages. The heterogeneous distribution of mycotoxins in food samples can affect the accuracy of results obtained with this approach. Duplicate diet studies could avoid sampling issues but requires suitable analytical methods for single or multi-mycotoxin determination and considerable commitment from the participants.

The measurement of specific urinary mycotoxin biomarkers is a valid alternative to measure exposure to mycotoxins providing that the excretion of biomarkers correlate well with mycotoxin intake. Suitable urinary biomarkers for AFB1, FB1, ZEA, DON and OTA are aflatoxin M1 (AFM1), FB1, ZEA $+\alpha$-zearalenol $(\alpha-Z O L)+\beta$-zearalenol $(\beta$-ZOL), DON + de-epoxydeoxynivalenol (DOM- 1$)$ and OTA, respectively. These biomarkers are excreted as free and conjugated forms therefore urine samples are usually digested with $\beta$-glucuronidase/sulfatase in order to deconjugate the conjugated forms and increase the concentration and detectability of free analytes. Human pilot studies and epidemiological studies based on biomarker approach have been performed for DON, AFB1, OTA and FB1. These studies were conducted by using analytical methods tailored for determination of biomarker(s) of a single mycotoxin [12-15]. LC-MS/MS is the ideal approach for simultaneous 
determination of analytes and its use for multi-biomarker determination in human and animal urine is recently increased $[8,16]$. In vivo experiments demonstrated a good correlation between the amount of mixtures of DON, OTA, ZEA, FB1 and AFB1 administered to piglets and the amount of relevant biomarkers excreted in $24 \mathrm{~h}$ post dose urine. Linear dose-response correlation coefficients ranged between 0.68 and 0.78 for the tested couples of mycotoxin/biomarker. Mean percentages of dietary mycotoxins excreted as biomarkers in $24 \mathrm{~h}$ post dose urine were $36.8 \%$ for ZEA, $28.5 \%$ for DON, $2.6 \%$ for FB1, 2.6\% for OTA and 2.5\% for AFB1 [17]. In this paper, we report the results on the occurrence of biomarkers to DON, OTA, ZEA, FB1 and AFB1 in urine samples of 52 volunteers resident in Apulia a region of Southern Italy.

\section{Results and Discussion}

The urinary concentrations of mycotoxin biomarkers could be very low for several reasons: (a) the levels of mycotoxins in foods and beverages can be very low especially for AFB1, OTA and ZEA; (b) the gastrointestinal absorption can be low as demonstrated for FB1, c) the serum half-life can be very high as demonstrated for OTA. The purification protocol used in our study for simultaneous determination of urinary DON, DOM-1, AFM1, FB1, ZEA, $\alpha$-ZOL, $\beta$-ZOL and OTA was successfully validated in a mini comparison study involving other laboratories that used either another multi-biomarker method or single-biomarker methods for determination of DON and FB1 [18]. To improve the sensitivity of the method we used an UPLC system coupled with a powerful and sensitive mass spectrometer (API $5000 \mathrm{MS} / \mathrm{MS}$ system with ESI interface). It was necessary to optimize the chromatographic conditions for optimal biomarker separation on Acquity BEH phenyl column as well as the MS/MS conditions. The optimized MS/MS conditions for each biomarker are reported in Table 1.

The main differences with the MS/MS parameters previously optimized on a QTrap system [19] were: (a) the increase in number of daughter ions from 3 to 4 for FB1, $\alpha$-ZOL and $\beta$-ZOL; (b) minor modification in the clustering potential, entrance potential, collision energy and collision cell exit potential (Table 1). The use of the Acquity BEH phenyl column and the development of a new linear gradient composition of the mobile phase permitted to reduce the run time from $46 \mathrm{~min}$ to $15 \mathrm{~min}$. However, each sample extract was analyzed twice in positive and negative mode. AFM1, FB1 and OTA were detected and measured in positive mode whereas DON, DOM-1, ZEA, $\alpha-Z O L$ and $\beta-Z O L$ were detected and measured in negative mode. The use of an UPLC system permitted to use a $150 \mathrm{~mm} \times 2.1 \mathrm{~mm}$ column with a $1.7 \mu \mathrm{m}$ particles size of stationary phase that produced sharp peak thus increasing peak high with consequent reduction of the limit of detection (LOD) and quantification (LOQ). The use of a powerful mass spectrometer (API $5000 \mathrm{MS} / \mathrm{MS}$ system) produced a further increase in method sensitivity. In particular, a marked increase in sensitivity (up to 114 times) was obtained, in descending order, for ZEA, $\beta-Z O L, \alpha-Z O L$, FB1, AFM1 and OTA with the new UPLC-MS/MS system as compared to the previous one (Table 2). No increase of sensitivity was obtained for DON whereas for DOM-1 an increase of LOQ value was observed probably due to ion suppression effect. 
Table 1. MS/MS conditions for detection of target analytes by MRM method.

\begin{tabular}{|c|c|c|c|c|c|c|c|}
\hline Analyte & Precursor ion & Q1 $(\mathbf{m} / \mathbf{z})$ & Q3 $(\mathbf{m} / \mathbf{z})$ & $\mathbf{D P}(\mathbf{V})$ & $\mathbf{E P}(\mathbf{V})$ & $\mathbf{C E}(\mathbf{V})$ & $\operatorname{CXP}(\mathrm{V})$ \\
\hline \multirow{3}{*}{ DON } & \multirow{3}{*}[\mathrm{DON}+\mathrm{CH}_{3}\mathrm{COO}]{$^{-}$} & \multirow{3}{*}{355.0} & 295.2 & \multirow{3}{*}{-50} & \multirow{3}{*}{-10} & -15 & \multirow{3}{*}{-20} \\
\hline & & & $265.0^{\mathrm{a}}$ & & & -22 & \\
\hline & & & $59.0^{\mathrm{a}}$ & & & -35 & \\
\hline \multirow{3}{*}{ DOM-1 } & \multirow{3}{*}[\mathrm{DOM}-1+\mathrm{CH}_{3}\mathrm{COO}]{$^{-}$} & \multirow{3}{*}{339.5} & 279.0 & -50 & & -12 & \multirow{3}{*}{-20} \\
\hline & & & 249.2 & -50 & -10 & -18 & \\
\hline & & & $59.0^{\mathrm{a}}$ & -55 & & -35 & \\
\hline \multirow{2}{*}{ AFM1 } & \multirow{2}{*}[\mathrm{AFM}1+\mathrm{H}]{$^{+}$} & \multirow{2}{*}{329.5} & $273.3^{\mathrm{a}}$ & \multirow{2}{*}{80} & \multirow{2}{*}{10} & 36.5 & \multirow{2}{*}{20} \\
\hline & & & $229.0^{\mathrm{a}}$ & & & 56 & \\
\hline \multirow{4}{*}{ FB1 } & \multirow{4}{*}[\mathrm{FB}1+\mathrm{H}]{$^{+}$} & \multirow{4}{*}{722.4} & $370.6^{\mathrm{a}}$ & \multirow{4}{*}{50} & \multirow{4}{*}{10} & 55 & \multirow{4}{*}{10} \\
\hline & & & $352.6^{\mathrm{a}}$ & & & 59 & \\
\hline & & & $334.7^{\mathrm{a}}$ & & & 65 & \\
\hline & & & $316.6^{\mathrm{a}}$ & & & 66 & \\
\hline \multirow{4}{*}{$\alpha-\mathrm{ZOL}$} & \multirow{4}{*}[\alpha-ZOL-H]{$^{-}$} & \multirow{4}{*}{319.1} & $188.2^{\mathrm{a}}$ & \multirow{4}{*}{-100} & \multirow{4}{*}{-10} & -38 & \multirow{4}{*}{-10} \\
\hline & & & $174.1^{\mathrm{a}}$ & & & -36 & \\
\hline & & & $160.1^{\mathrm{a}}$ & & & -43 & \\
\hline & & & $130.0^{\mathrm{a}}$ & & & -48 & \\
\hline \multirow{4}{*}{$\beta-\mathrm{ZOL}$} & \multirow{4}{*}[\beta-\mathrm{ZOL}-\mathrm{H}]{$^{-}$} & \multirow{4}{*}{319.1} & $188.2^{\mathrm{a}}$ & \multirow{4}{*}{-100} & & -38 & \\
\hline & & & $174.1^{\mathrm{a}}$ & & & -36 & 10 \\
\hline & & & $160.1^{\mathrm{a}}$ & & -10 & -43 & -10 \\
\hline & & & $130.0^{\mathrm{a}}$ & & & -48 & \\
\hline & & & 273.3 & & & -29 & \\
\hline ZEA & {$[\mathrm{ZEA}-\mathrm{H}]^{-}$} & 317.2 & 175.0 & -100 & -10 & -35 & -10 \\
\hline & & & $131.0^{\mathrm{a}}$ & & & -40 & \\
\hline & & & 358.5 & & & 30 & \\
\hline OTA & {$[\mathrm{OTA}+\mathrm{H}]^{+}$} & 404.2 & 257.3 & 90 & 10 & 38 & 10 \\
\hline & & & $239.2^{\mathrm{a}}$ & & & 47 & \\
\hline
\end{tabular}

Notes: ${ }^{a}$ Transitions used for quantitation; $Q 1$ : first quadrupole; $Q 3$ : third quadrupole; $D P$ : declustering potential; $E P$ : entrance potential; $C E$. collision energy; $C X P$. collision cell exit potential

Table 2. LOQ values for DON, AFM1, FB1, $\beta-Z O L, \alpha-Z O L$, ZEA and OTA in human urine samples obtained with two different LC-MS/MS systems: LC-QTrap MS/MS system and UPLC-API $5000 \mathrm{MS} / \mathrm{MS}$ system.

\begin{tabular}{ccc}
\hline \multirow{2}{*}{ Biomarker } & $\begin{array}{c}\text { Apparatus 1: LC-QTrap MS/MS } \\
\text { LOQ }(\mathbf{n g} / \mathbf{m L})\end{array}$ & $\begin{array}{c}\text { Apparatus 2: UPLC-API 5000 MS/MS } \\
\text { LOQ }(\mathbf{n g} / \mathbf{m L})\end{array}$ \\
\hline ZEA & 0.8 & 0.007 \\
$\beta$-ZOL & 4.4 & 0.054 \\
$\alpha$-ZOL & 1.6 & 0.030 \\
FB1 & 0.1 & 0.010 \\
AFM1 & 0.1 & 0.020 \\
OTA & 0.02 & 0.006 \\
DON & 1.5 & 1.5 \\
DOM-1 & 1.5 & 9.9 \\
\hline
\end{tabular}


In Figure 1 are reported three chromatograms of a naturally contaminated urine sample containing DON, ZEA, $\alpha$-ZOL, $\beta$-ZOL, FB1 and OTA.

Figure 1. LC-MS/MS chromatograms obtained in negative ion mode $(\mathbf{a}, \mathbf{b})$ and in positive ion mode (c) of a naturally urine sample (\#4) containing $11.33 \mathrm{ng} / \mathrm{mL}$ of DON, $0.108 \mathrm{ng} / \mathrm{mL}$ of $\beta$-ZOL, $0.123 \mathrm{ng} / \mathrm{mL}$ of $\alpha-Z O L, 0.082 \mathrm{ng} / \mathrm{mL}$ of ZEA, $0.26 \mathrm{ng} / \mathrm{mL}$ of $\mathrm{FB} 1$ and $0.06 \mathrm{ng} / \mathrm{mL}$ of OTA.

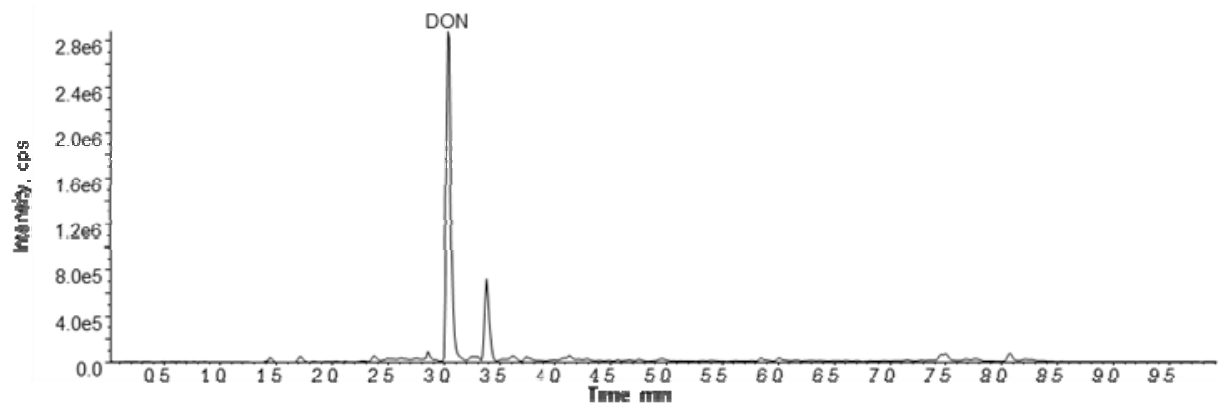

(a)

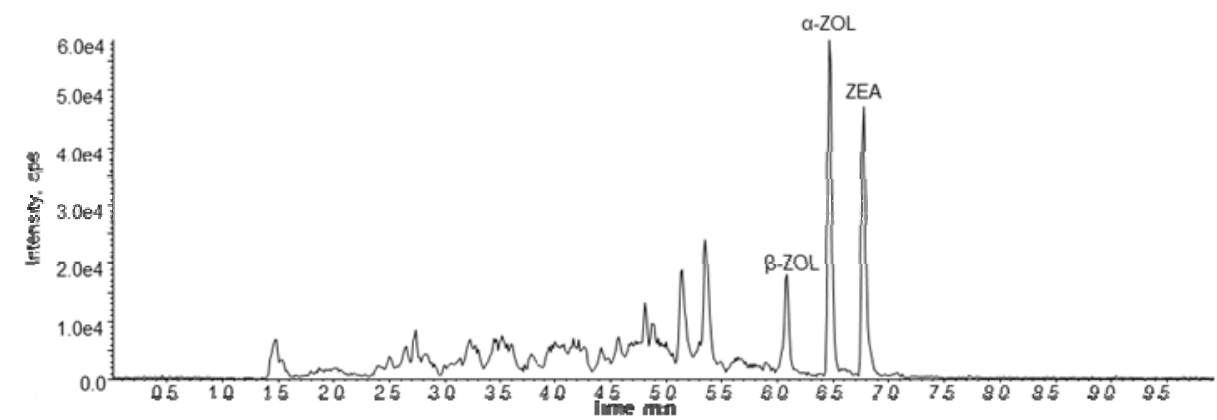

(b)

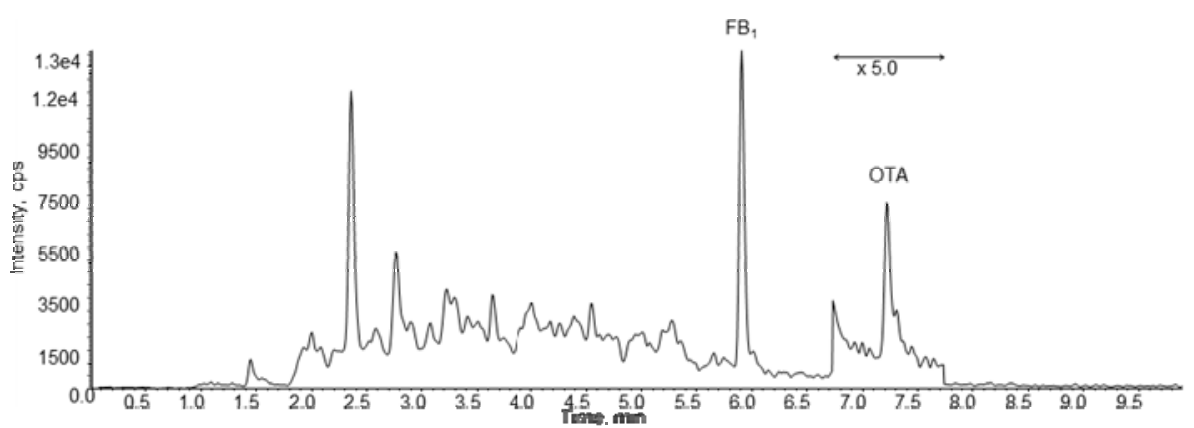

(c)

A summary of the results of the urine samples collected in this study are reported in Table 3. All urine samples contained ZEA, $\alpha-Z O L$ and OTA whereas $\beta-Z O L$ was found in $98 \%$ of samples. DON, FB1 and AFM1 were found in $96 \%, 56 \%$ and $6 \%$ of urine samples, respectively. The highest mean biomarker concentration was found for DON $(11.89 \mathrm{ng} / \mathrm{mL})$ followed by OTA $(0.144 \mathrm{ng} / \mathrm{mL}), \beta-Z O L$ (0.090 $\mathrm{ng} / \mathrm{mL}), \alpha$-ZOL $(0.077 \mathrm{ng} / \mathrm{mL})$, AFM1 $(0.068 \mathrm{ng} / \mathrm{mL})$, ZEA $(0.057 \mathrm{ng} / \mathrm{mL})$ and FB1 $(0.055 \mathrm{ng} / \mathrm{mL})$. The highest individual biomarker concentration was measured for DON $(67.36 \mathrm{ng} / \mathrm{mL})$ followed by OTA $(2.129 \mathrm{ng} / \mathrm{mL})$, FB1 $(0.352 \mathrm{ng} / \mathrm{mL}), \alpha$-ZOL $(0.176 \mathrm{ng} / \mathrm{mL}), \beta-Z O L(0.135 \mathrm{ng} / \mathrm{mL})$, AFM1 $(0.146 \mathrm{ng} / \mathrm{mL})$ and ZEA $(0.120 \mathrm{ng} / \mathrm{mL})$. From this Table it is evident that urinary 
concentrations of DON are much higher to those of the other biomarkers. Moreover, the highest inhomogeneous distributions of concentrations was observed for OTA as demonstrated by the value of relative standard deviation (RSD) of the mean (217\%) followed by FB1 (133\%), AFM1 (99\%), DON (84\%), ZEA (40\%), $\alpha$-ZOL (35\%) and $\beta$-ZOL (16\%).

Table 3. Results of mycotoxin biomarkers in human urine samples collected in Southern Italy and estimated values of PDI for each mycotoxin

\begin{tabular}{|c|c|c|c|c|c|c|c|}
\hline Biomarkers & DON & $\beta-Z O L$ & $\alpha-Z O L$ & ZEA & FB1 & OTA & AFM1 \\
\hline N. positive (\%) & $50(96)$ & $51(98)$ & $52(100)$ & $52(100)$ & $29(56)$ & $52(100)$ & $3(6)$ \\
\hline Mean, ng/mL & 11.89 & 0.090 & 0.077 & 0.057 & 0.055 & 0.144 & 0.068 \\
\hline $\mathrm{SD}, \mathrm{ng} / \mathrm{mL}$ & 10.05 & 0.014 & 0.027 & 0.023 & 0.073 & 0.312 & 0.067 \\
\hline Median, ng/mL & 10.32 & 0.088 & 0.074 & 0.056 & 0.029 & 0.061 & 0.10 \\
\hline Max, ng/mL & 67.36 & 0.135 & 0.176 & 0.120 & 0.352 & 2.129 & 0.146 \\
\hline Mycotoxins & DON & & ZEA & & FB1 & OTA & AFB1 \\
\hline $\begin{array}{c}\text { Mean } \mathrm{PDI}^{\mathrm{a}}, \mu \mathrm{g} / \mathrm{kg} \\
\text { body weight }\end{array}$ & 1.03 & & 0.015 & & 0.053 & 0.139 & 0.068 \\
\hline $\begin{array}{c}\text { Max } \mathrm{PDI}^{\mathrm{a}}, \mu \mathrm{g} / \mathrm{kg} \\
\text { body weight }\end{array}$ & 5.90 & & 0.029 & & 0.338 & 2.047 & 0.142 \\
\hline $\begin{array}{l}\% \text { of } \mathrm{PDI}^{\mathrm{a}} \text { values } \\
\text { exceeding the TDI }\end{array}$ & 40 & & 0 & & 0 & 94 & 0 \\
\hline $\begin{array}{c}\text { Mean } \mathrm{PDI}^{\mathrm{b}}, \mu \mathrm{g} / \mathrm{kg} \\
\text { body weight }\end{array}$ & 0.59 & & $--^{c}$ & & 0.274 & $--^{c}$ & $--^{\mathrm{c}}$ \\
\hline $\begin{array}{l}\text { Max } \mathrm{PDI}^{\mathrm{b}}, \mu \mathrm{g} / \mathrm{kg} \\
\text { body weight }\end{array}$ & 3.37 & & $--^{c}$ & & 1.759 & $--^{c}$ & $--^{c}$ \\
\hline $\begin{array}{l}\% \text { of } \mathrm{PDI}^{\mathrm{b}} \text { values } \\
\text { exceeding the TDI }\end{array}$ & 6 & & $--^{c}$ & & 0 & $--^{c}$ & $--^{c}$ \\
\hline $\begin{array}{c}\mathrm{TDI}^{\mathrm{d}}, \mu \mathrm{g} / \mathrm{kg} \text { body } \\
\text { weight }\end{array}$ & 1.0 & & 0.2 & & 2.0 & 0.017 & $--^{\mathrm{e}}$ \\
\hline
\end{tabular}

Notes: ${ }^{a}$ calculated based on piglet excretion data; ${ }^{b}$ calculated based on human excretion data $(50 \%$ for DON, $0.5 \%$ for FB1) reported in Shephard et al. [8]; ${ }^{c}$ not estimated due to unavailability of human excretion rate;

${ }^{\mathrm{d}}$ TDI values are reported in [19] and references therein; ${ }^{\mathrm{e}}$ there is no TDI value for AFB1 because it is a carcinogenic mycotoxin.

The results of the co-occurrence of multiple biomarkers in the tested urine samples are reported in Table 4 . The majority of urine samples (52\%) contained biomarkers of DON, ZEA, FB1 and OTA whereas $38 \%$ of samples contained biomarkers of DON, ZEA and OTA. The co-occurrence of biomarkers of all mycotoxins was found in two urine samples. Moreover, no individual was found unexposed or exposed to a single mycotoxin since all investigated urine samples contained biomarkers of at least two mycotoxins. No important differences were observed for the results obtained for male and female individuals with the exception that the two urine samples containing biomarkers of all mycotoxins were from females. Mixtures of biomarkers of DON, ZEA, FB1 and OTA were found in human urine samples collected in the former Transkei, South Africa [8]. Co-occurrence of biomarkers of 2 to 5 mycotoxins was reported in human urine samples collected in Cameroon [20,21]. 
Table 4. Incidence of individuals exposed to mixtures of mycotoxins for a total of 52 volunteers (26 males and 26 females).

\begin{tabular}{ccc}
\hline Multiple mycotoxins exposure & n. positive samples & \% of positive samples \\
\hline DON, ZEA, FB1, OTA, AFB1 & 2 & 4 \\
DON, ZEA, FB1, OTA & 27 & 52 \\
DON, ZEA, OTA & 20 & 38 \\
DON, ZEA, OTA, AFB1 & 1 & 2 \\
ZEA, OTA & 2 & 4 \\
TOTAL & 52 & 100 \\
\hline
\end{tabular}

This is the first report on the simultaneous detection of biomarkers of the 5 principal mycotoxins in Italy. The simultaneous presence of DON and OTA in human urine in Italy was previously reported [19] whereas the presence of ZEA, $\alpha-Z O L, \beta-Z O L, F B 1$ and AFM1 is reported herein for the first time. The presence of DON and OTA in almost all urinary samples is not surprising because these mycotoxins are usually found in cereals and derived products, staple foods of Italian people [22,23]. Interestingly, $56 \%$ of urine samples contained FB1, a mycotoxin mainly found in maize and deriving products. Although these products are not staple foods in Italy they are widely consumed as chips, polenta, popcorn, beer, cornflakes, snacks, muesli and mixed cereals [22,24]. The consumption of these products could explain the high percentage of urine samples containing FB1. The low concentrations of biomarkers of ZEA in all tested urine samples is also a new and interesting information and demonstrate that all the 52 volunteers that participated in our study were exposed to low levels of ZEA. On the other hand the results of a large survey conducted in Europe showed that only 32\% of 5018 samples of cereals and derived products, the main source for ZEA exposure, were found contaminated with this mycotoxin [22]. A possible explanation of this apparent paradox could be that the high sensitivity of the multi biomarker method used in the present study can detect urinary biomarker deriving from the consumption of foods contaminated with very low levels of ZEA that could not be detected with conventional analytical methods. The presence of AFM1 in only 3 urine samples demonstrates that human exposure to AFB1 is quite limited in this area of Southern Italy. In fact ground nuts and tree nuts, the main source of human AFB1 exposure in Italy, are occasionally consumed in this Country. The absence of AFM1 in almost all urine samples suggests that maize and derived products consumed in Southern Italy are probably not contaminated with AFB1 although it is well known that maize is a major source of AFB1 in some countries. The absence of AFM1 in human urine and AFB1 in maize based food was also reported in South Africa [8].

The urinary biomarker concentrations measured in this study were used to estimate the probable daily intake (PDI) of each mycotoxin by each volunteer according to Equation (1).

$$
\text { PDI }=C \times \frac{V}{W} \times \frac{100}{E}
$$

PDI probable daily intake of mycotoxin ( $\mu \mathrm{g} / \mathrm{kg}$ body weight);

$C$ human urinary biomarker concentration $(\mu \mathrm{g} / \mathrm{L})$;

$V$ mean 24 h human urine volume $(1.5 \mathrm{~L})$;

$W$ mean human body weight $(60 \mathrm{~kg})$;

$E$ mean urinary excretion rate of mycotoxin in $24 \mathrm{~h}$ post dose in piglets (36.8\% for ZEA, $27.9 \%$ for DON, 2.6\% for FB1, 2.6\% for OTA and 2.5\% for AFM1 [17]). 
Based on mean urinary concentration of each mycotoxin biomarker measured in the 52 human urine samples and the mean urinary excretion rate in piglets for each mycotoxin, the estimated daily mean intake of the 5 investigated mycotoxins were calculated and are reported in Table 3 . Due to the unavailability of human excretion rate for all the 5 mycotoxins considered in this study we used the $24 \mathrm{~h}$ excretion rate measured in piglets [17] to estimate the PDI in human. However, since sufficient data of human excretion rate of DON and FB1 have been reported [8], additional values of PDI, calculated with human data, were added in Table 3. In this table, the values of max PDI for each mycotoxin and the percentage of individuals that exceeded the tolerable daily intake (TDI) for each mycotoxin are also reported. The estimated mean values of PDI were below or equal to the TDI or provisional maximum TDI (PMTDI) for DON, FB1 and ZEA. Individual analysis of PDI values obtained for DON revealed that $40 \%$ of volunteers exceeded the value of TDI of $1 \mu \mathrm{g} / \mathrm{kg}$ body weight established for this mycotoxin with a maximum value of PDI of $5.90 \mu \mathrm{g} / \mathrm{kg}$ body weight. Previous studies conducted in UK, France and Sweden using the urinary DON concentration to estimate DON exposure reported mean values of PDI of $0.12-0.73,0.61$ and $0.16 \mu \mathrm{g} / \mathrm{kg}$ body weight, respectively [25-30]. Human exposure to DON in our study seems to be higher to that estimated in UK since both mean values of PDI and $\%$ of individuals that exceed TDI are higher as compared to UK where some $5 \%$ of the adult population may exceed the TDI for DON intake [25]. The mean PDI values of DON derived from food analyses in UK $(0.14-0.23 \mu \mathrm{g} / \mathrm{kg}$ body weight) seems to be slightly lower as compared to PDI values estimated from urinary DON in that Country [25-28,31]. The mean PDI values of DON derived from food analysis in Europe and reported by SCOOP report [22] and FAO/WHO [32] were 0.34 and $1.4 \mu \mathrm{g} / \mathrm{kg}$ body weight, respectively. The results of our study obtained with the biomarker approach fall within this interval and is more close to the PDI reported by FAO/WHO [32]. A lower mean value of PDI $(0.59 \mu \mathrm{g} / \mathrm{kg}$ body weight) was obtained by using the human excretion rate of 50\% reported by Shephard et al. [8] as well as the percentage of individuals $(6 \%)$ that exceeded the value of TDI for this mycotoxin.

The mean value of PDI for FB1 calculated in our study with biomarker approach $(0.053 \mu \mathrm{g} / \mathrm{kg}$ body weight $)$ is far below the TDI established for this mycotoxin $(2 \mu \mathrm{g} / \mathrm{kg}$ body weight $)$ and all individual values are below the TDI for this mycotoxin with a maximum value of PDI of $0.338 \mu \mathrm{g} / \mathrm{kg}$ body weight (Table 3). These results can only be compared with results obtained in Guatemala, South Africa and Mexico because urinary FB1 in European population has not been performed yet. The mean values of PDI estimated with biomarker approach in Guatemala, Mexico and South Africa were 0.45, 0.37 and $0.22 \mu \mathrm{g} / \mathrm{kg}$ body weight, respectively $[15,33,34]$. Comparison with these data shows that in our study human exposure to FB1 is about 10 times lower to those estimated in Guatemala, Mexico and South Africa. The mean PDI values of FB1 obtained in our study with biomarker approach $(0.053 \mu \mathrm{g} / \mathrm{kg}$ body weight) is quite similar to the PDI of $0.056 \mu \mathrm{g} / \mathrm{kg}$ body weight estimated with diet approach by Brera et al. in Italy [24]. The estimated mean PDI value reported by FAO/WHO [32] for Europe with the diet approach was $0.2 \mu \mathrm{g} / \mathrm{kg}$ body weight which is 3.8 times higher than the intake estimated in our study. A much higher mean value of PDI $(0.274 \mu \mathrm{g} / \mathrm{kg}$ body weight $)$ was obtained by using the human excretion rate of $0.5 \%$ reported by Shephard et al., [8]. However even with this estimate all individuals resulted below the value of TDI for this mycotoxin.

The mean value of PDI of ZEA estimated with biomarker approach in our study $(0.015 \mu \mathrm{g} / \mathrm{kg}$ body weight $)$ is about ten times below the TDI established for this mycotoxin $(0.2 \mu \mathrm{g} / \mathrm{kg}$ body weight). The maximum estimated value of PDI is $0.029 \mu \mathrm{g} / \mathrm{kg}$ body weight (Table 3). In Italy, the mean value of 
PDI estimated with the diet approach was $0.0008 \mu \mathrm{g} / \mathrm{kg}$ body weight [22] whereas the PDI reported by EFSA for Europe was $0.03-0.06 \mu \mathrm{g} / \mathrm{kg}$ body weight [35]. The mean value of PDI estimated in our study is higher than the Italian value reported in the SCOOP report and lower than the lower limit of the range reported by EFSA [35].

The occurrence of ZEA biomarkers in human urine was recently reported in USA and Cameroon. Mixtures of ZEA, $\alpha-Z O L$ and $\beta$-ZOL were detected and measured in 55\% of urine samples collected from girls resident in New Jersey with mean concentrations ranging from $0.35 \mathrm{ng} / \mathrm{mL}$ ( $\beta-Z O L)$ to $1.82 \mathrm{ng} / \mathrm{mL}$ (ZEA) [36]. These concentrations are 4-32 times higher the mean concentrations found in our study for these ZEA biomarkers which means that in New Jersey human exposure to ZEA is higher as compared to Italy. Mixtures of ZEA, $\alpha$-ZOL and ZEA-glucuronide were found in a low percentage (5\%) of human urine samples collected in Cameroon with a total mean concentration of $0.74 \mathrm{ng} / \mathrm{mL}$ [20].

As reported in Table 3 the mean value of PDI of OTA estimated with biomarker approach in our study $(0.139 \mu \mathrm{g} / \mathrm{kg}$ body weight $)$ is about 8 times higher than the TDI $(0.017 \mu \mathrm{g} / \mathrm{kg}$ body weight $)$ established for this mycotoxin [37]. The maximum value of PDI estimated in our study $(2.047 \mu \mathrm{g} / \mathrm{kg}$ body weight) is 147 times higher than the TDI and $94 \%$ of individuals participating in our study exceeded the value of TDI (Table 3). Several studies reported the occurrence of OTA in human urine with a high percentage of positive samples but the relevant values of PDI from these results were not calculated nor reported. We used the human urinary concentrations of OTA reported in literature to estimate values of PDI of OTA by using the Equation (1) reported above. Urinary concentrations of OTA reported by Fazekas et al. [38] and Duarte et al. [14,39] produced values of PDI equal or below the TDI, whereas from the results of Pena et al. [40], Manique et al. [41], Coronel et al. [42], Gilbert et al. [43] and Domijan et al. [44] the estimated values of PDI were higher the TDI. Our result of estimated mean PDI, though higher than the TDI, is lower than the mean PDI values estimated from the urinary concentrations reported by Domijan et al. in Croatia [44] and Coronel et al. in Spain [42]. When compared to the PDI values estimated with urinary concentrations reported by Fazekas et al. [38], Duarte et al. [14,39], Pena et al. [40], Manique et al. [41] and Gilbert et al. [43] our estimated PDI values were higher.

In Europe, the mean values of PDI of OTA estimated with the diet approach range from 0.0011 to $0.024 \mu \mathrm{g} / \mathrm{kg}$ body weight [23]. The mean value of PDI estimated with our biomarker study $(0.139 \mu \mathrm{g} / \mathrm{kg}$ body weight $)$ is $5.8-127$ times higher than the European PDI values estimated with diet approach. All together, these data clearly show that the estimated human exposure to OTA is higher when using the biomarker approach as compared to the diet approach. Possible explanations of these differences could be: a) the urinary excretion rate of OTA in piglets, used in this study to estimate PDI values, is completely different from that in human, b) the food diet approach did not cover all sources of OTA exposure due to the occurrence of this mycotoxin in a very high number of different foods and beverages. It is well known that OTA is the mycotoxin more widespread in several different types of foods and beverages $[23,32]$.

The results shown in Table 3 indicate that AFM1 was detected and measured in only 3 urine samples ( $6 \%$ of total samples) which confirm that human exposure to AFB1 was sporadic. The relevant mean value of PDI of AFB1, calculated for the 3 positive subjects, is $0.068 \mu \mathrm{g} / \mathrm{kg}$ body weight. As reported above, there is no TDI value for AFB1 because this is a carcinogenic mycotoxin. The incidence of positive samples and mean urinary AFM1 concentration measured in positive 
samples in our study are comparable to those reported by Hatem et al. from Marasmus, Egypt [45]. Polychronaki et al. reported, for Guinea, a mean urinary concentration of AFM1 in positive samples similar to those found in our study but a higher percentage of positive samples (64\%) [13]. The mean value of PDI of AFB1 estimated with biomarker approach for the three volunteers participating in our study $(0.068 \mu \mathrm{g} / \mathrm{kg}$ body weight) is largely higher than the PDI of aflatoxin intake measured with the diet approach in Europe (0.00016-0.00055 $\mu \mathrm{g} / \mathrm{kg}$ body weight) [23].

\section{Experimental Section}

\subsection{Chemicals and Reagents}

Standard solutions were purchased from Romer Labs Diagnostic (Tulln, Austria). In particular, solutions of DON $(100 \mu \mathrm{g} / \mathrm{mL})$, DOM-1 $(50 \mu \mathrm{g} / \mathrm{mL})$, AFM1 $(0.5 \mu \mathrm{g} / \mathrm{mL})$, ZEA $(100 \mu \mathrm{g} / \mathrm{mL}), \alpha-Z O L$ $(10 \mu \mathrm{g} / \mathrm{mL}), \beta-Z O L(10 \mu \mathrm{g} / \mathrm{mL})$ and OTA $(10 \mu \mathrm{g} / \mathrm{mL})$ were prepared in acetonitrile $(\mathrm{ACN})$ whereas FB1 solution $(50 \mu \mathrm{g} / \mathrm{mL})$ were prepared in acetonitrile-water (50:50). $\beta$-glucuronidase/sulfatase type H-2 from Helix pomatia (specific activity 130,200 units/mL $\beta$-glucuronidase, 709 units $/ \mathrm{mL}$ sulfatase). Chromatography-grade methanol $(\mathrm{MeOH})$ and glacial acetic acid were obtained from Carlo Erba (Milan, Italy). Ultrapure water was produced by use of a Milli-Q system (Millipore, Bedford, MA, USA). Myco6in ${ }^{\circledR}$ immunoaffinity columns were purchased from Vicam L.P (Watertown, MA, USA). OASIS ${ }^{\circledR}$ HLB columns, $60 \mathrm{mg}, 3 \mathrm{~mL}$ were purchased from Waters (Milford, MA, USA) and regenerated cellulose filters $(0.45 \mu \mathrm{m})$ were purchased from Sartorius Stedim Biotech (Goettingen, Germany).

\subsection{Equipment and Conditions}

LC-MS/MS analyses were performed on a triple quadrupole API 5000 system (Applied Biosystems, Foster City, CA, USA), equipped with a ESI interface and an Acquity UPLC system comprising a binary pump and a microautosampler from Waters (Milford, MA, USA). The analytical column was an Acquity UPLC BEH phenyl column $(2.1 \mathrm{~mm} \times 150 \mathrm{~mm}, 1.7 \mu \mathrm{m}$ particles; Waters $)$. The column oven was set at $40{ }^{\circ} \mathrm{C}$. The flow rate of the mobile phase was $250 \mu \mathrm{L} / \mathrm{min}$ and the injection volume was $10 \mu \mathrm{L}$. For multi-biomarker separation a multiple linear binary linear gradient of acidic $\mathrm{MeOH}$ (containing $0.5 \%$ acetic acid) in water (containing $0.5 \%$ acetic acid) was developed and used as mobile phase as follows: from $20 \%$ to $80 \% \mathrm{MeOH}$ in $5 \mathrm{~min}$, then maintained at $80 \% \mathrm{MeOH}$ for $5 \mathrm{~min}$, then brought to $20 \% \mathrm{MeOH}$ in $0.5 \mathrm{~min}$ and left to equilibrate for $4.5 \mathrm{~min}$ before the next run. For LC-MS/MS analyses, the ESI interface was used in positive ion mode for AFM1, FB1 and OTA and in negative ion mode for DON, DOM-1, ZEA, $\alpha$-ZOL and $\beta$-ZOL. The mass spectrometer operated in MRM (multiple reaction monitoring) mode and the optimized MS/MS conditions for each analyte are listed in Table 1. In particular, 4 transitions were monitored for confirmation of FB1, $\alpha-Z O L$ and $\beta$-ZOL whereas the sum of the 4 transitions were used for quantification; for DON 3 transitions for confirmation and the sum of 2 transition for quantification; for DOM-1, ZEA and OTA 3 transitions for confirmation and 1 transition for quantification; for AFM1 2 transitions for confirmation and the sum of 2 transition for quantification. Interface conditions were: TEM, $450{ }^{\circ} \mathrm{C}$; CUR, nitrogen, 20 psi; GS1, air, 60 psi; GS2, air, 40 psi; ionspray voltage $+5500 \mathrm{~V}$ or $-4500 \mathrm{~V}$. The signal of each compound was preliminary optimized with each proposed ionization condition. The tuning procedure included the 
optimization of source parameters during infusion of $1 \mu \mathrm{g} / \mathrm{mL}$ standard solution $(0.1 \mu \mathrm{g} / \mathrm{mL}$ for AFM1) of the individual toxins in $\mathrm{MeOH}$-water (20:80) containing $0.5 \%$ acetic acid at $10 \mu \mathrm{L} / \mathrm{min}$, using an Harvard 11 plus infusion pump, into the UPLC mobile phase (50/50 water/methanol at $250 \mu \mathrm{L} / \mathrm{min}$ ) by means of a minimum dead volume T-piece connected after the analytical column.

\subsection{Participants and Urine Collection}

For the evaluation of human exposure to DON, AFB1, FB1, ZEA and OTA 100 individuals residing in 10 municipalities of Puglia region (Southern Italy) were invited to participate in the urine sampling. Each individual was asked to provide a first morning urine sample and to fill out a questionnaire concerning age, gender and health status. The majority of individuals gave oral informed consent and donated a sample of first morning urine and the filled in questionnaire. Fifty-two individuals (participation rate 52\%), 26 males and 26 females, (mean age 41 years, range 3-85 years) were recruited from municipalities of Bari, Triggiano, Mola di Bari, Monopoli, Adelfia, Conversano, Polignano a Mare, Bitonto, Martina Franca and Statte. The remaining 48 individuals failed to donate their morning urine samples. All participants collected their urine samples on 26 April 2011. Urine samples were stored at $-18{ }^{\circ} \mathrm{C}$ until analysis for identification and determination of DON, DOM-1, AFM1, FB1, ZEA, $\alpha-Z O L, \beta-Z O L$ and OTA. Eleven volunteers declared to have health problems, in particular three individuals with hypertension, three with allergies, three with diabetes, one with hyperthyroidism and one at risk of thrombosis.

\subsection{Calibration Curves}

A mixed standard solution with a final concentration of $150 \mathrm{ng} / \mathrm{mL}$ DON, $20 \mathrm{ng} / \mathrm{mL}$ of DOM-1, $1.8 \mathrm{ng} / \mathrm{mL}$ AFM1, $28 \mathrm{ng} / \mathrm{mL}$ FB1, $35 \mathrm{ng} / \mathrm{mL} \beta$-ZOL, $23 \mathrm{ng} / \mathrm{mL} \alpha-Z O L, 12 \mathrm{ng} / \mathrm{mL}$ ZEA and $2 \mathrm{ng} / \mathrm{mL}$ OTA was prepared by mixing appropriate volumes of commercially available standard solutions and appropriate volume of ACN. Five standard calibration solutions covering appropriate range of analyte concentrations were prepared by portioning adequate volumes of mixed standard solution that were dried and reconstituted with $200 \mu \mathrm{L}$ of initial LC-MS/MS mobile phase. In particular, the 5 standard calibration solutions were prepared by drying $25,125,250,375$ and $1000 \mu \mathrm{L}$ of mixed standard solution that were reconstituted with $200 \mu \mathrm{L}$ of initial LC-MS/MS mobile phase. Matrix-matched calibration solutions were prepared in 5 purified urinary extracts. In particular, urine from 6 individuals were pooled and mixed then 5 aliquots of $6 \mathrm{~mL}$ each were purified according to the protocol reported above. The 5 eluates collected from OASIS ${ }^{\circledR}$ HLB and Myco6in $1{ }^{\circledR}$ columns were spiked with 5 aliquots of the mixed standard solution $(25,125,250,375$ and $1,000 \mu \mathrm{L})$, dried and reconstituted with $200 \mu \mathrm{L}$ of initial LC-MS/MS mobile phase. The ranges of mycotoxin concentrations in the calibration solutions were, therefore: $18.75-750.00 \mathrm{ng} / \mathrm{mL}$ for DON, $2.50-100.00 \mathrm{ng} / \mathrm{mL}$ for DOM-1, $0.22-8.76 \mathrm{ng} / \mathrm{mL}$ for AFM1, 3.50-140.00 ng/mL for FB1, 4.38-175.00 ng/mL for $\beta-Z O L, ~ 2.88-115.00 \mathrm{ng} / \mathrm{mL}$ for $\alpha-Z O L$, $1.50-60.00 \mathrm{ng} / \mathrm{mL}$ for ZEA and $0.25-10.00 \mathrm{ng} / \mathrm{mL}$ for OTA. 


\subsection{Analysis of Urinary Biomarkers}

Urine samples were hydrolyzed with $\beta$-glucuronidase/sulfatase enzyme to hydrolyse glucuronide and/or sulfate conjugates of DON, DOM-1, ZEA, $\alpha-Z O L, \beta-Z O L$ and then purified with a multi-antibody and OASIS ${ }^{\circledR}$ HLB columns according to the protocol reported elsewhere [19]. In brief, a $6 \mathrm{~mL}$ urine sample was hydrolyzed at $37{ }^{\circ} \mathrm{C}$ for $18 \mathrm{~h}$ with $300 \mu \mathrm{L}$ of $\beta$-glucuronidase/sulfatase type H-2 from Helix pomatia. Hydrolyzed sample was diluted with $6 \mathrm{~mL}$ of water and purified on a Myco6in $1^{\circledR}$ and OASIS ${ }^{\circledR}$ HLB columns connected in tandem. The OASIS ${ }^{\circledR}$ HLB column was previously conditioned by passing $2 \mathrm{~mL} \mathrm{MeOH}$ and $2 \mathrm{~mL}$ ultrapure water. After sample application and elution, the two columns were separated, the Myco6in $1^{\circledR}$ was washed with water $(4 \mathrm{~mL})$ and eluted with methanol $(3 \mathrm{~mL})$ and water $(2 \mathrm{~mL})$ that were collected in a vial. The OASIS ${ }^{\circledR}$ HLB column was washed with methanol/water $(20: 80,1 \mathrm{~mL})$ and DON that had passed through the Myco6in $1^{\circledR}$ and retained on the OASIS ${ }^{\circledR}$ HLB was eluted with methanol/water $(40: 60,1 \mathrm{~mL})$. The separate eluates from the two columns were combined, dried down and reconstituted in methanol/water $(20: 80,200 \mu \mathrm{L})$ with $0.5 \%$ acetic acid. Purified extract was filtered through a regenerated cellulose filter and a volume of $10 \mu \mathrm{L}$ (equivalent to $0.3 \mathrm{~mL}$ urine) was analyzed by UPLC-MS/MS.

\section{Conclusions}

The improved UPLC-MS/MS method for simultaneous determination of urinary biomarker for DON, FB1, OTA, AFB1 and ZEA was suitable to detect and accurately measure the low mycotoxin biomarker concentrations naturally occurring in the human urine samples collected in this study.

A multiple mycotoxin exposure was found for all tested volunteers participating in the study.

This is the first report on the occurrence of urinary AFM1, FB1, ZEA and ZOLs in Italy.

The estimated PDI values of OTA largely exceeded the TDI value for this mycotoxin in 94\% of volunteers.

The mean estimated PDI of DON is similar to the TDI value for this mycotoxin but in $40 \%$ of volunteers it exceeded the value of TDI.

The values of PDI estimated herein with urinary biomarker approach matched quite well with the intake estimated with the diet approach reported in the literature for DON, FB1 and ZEA whereas for OTA and AFB1 the intake estimated with the biomarker approach was much higher than the intake estimated with the diet approach reported in the literature.

\section{Acknowledgments}

This work was supported by the EU-FP7 MYCORED project (grant agreement no. 222690). We thank Water Research Institute, IRSA-CNR, Bari that made available the API 5000 UPLC-MS/MS system and Vito Locaputo for valuable assistance during the LC-MS/MS analyses.

\section{Conflicts of Interest}

The authors declare no conflict of interest. 


\section{References}

1. Shephard, G.S.; Berthiller, F.; Burdaspal, P.; Crews, C.; Jonker, M.A.; Krska, R.; Lattanzio, V.M.T.; MacDonald, S.; Malone, R.J.; Maragos, C.; et al. Developments in mycotoxin analysis: An update for 2011-2012. World Mycotoxin J. 2013, 6, 3-30.

2. Shephard, G.S.; Berthiller, F.; Burdaspal, P.; Crews, C.; Jonker, M.A.; Krska, R.; MacDonald, S.; Malone, R.J.; Maragos, C.; Sabino, M.; et al. Developments in mycotoxin analysis: An update for 2010-2011. World Mycotoxin J. 2012, 5, 3-30.

3. Serrano, A.B.; Font, G.; Ruiz, M.J.; Ferrer, E. Co-occurrence and risk assessment of mycotoxins in food and diet from Mediterranean area. Food Chem. 2012, 135, 423-429.

4. Lindblad, M.; Gidlund, A.; Sulyok, M.; Börjesson, T.; Krska, R.; Olsen, M.; Fredlund, E. Deoxynivalenol and other selected Fusarium toxins in Swedish wheat- Occurrence and correlation to specific Fusarium. species. Int. J. Food Microbiol. 2013, 167, 284-291.

5. Ibáñez-Vea, M.; Martínez, R.; Gonzáles-Peñas, E.; Lizarraga, E.; López de Cerain, A. Co-occurrence of aflatoxins, ochratoxin A and zearalenone in breakfast cereals from Spanish market. Food Control 2011, 22, 1949-1955.

6. Njobeh, P.B.; Dutton, M.F.; Koch, S.H.; Chuturgoon, A.A.; Stoev, S.D.; Mosonik, J.S. Simultaneous occurrence of mycotoxins in human food commodities from Cameroon. Mycotoxin Res. 2010, 26, 47-57.

7. Shephard, G.S.; Burger, H.M.; Gambacorta, L.; Krska, R.; Powers, S.P.; Rheeder, J.P.; Solfrizzo, M.; Sulyok, M.; Visconti, A.; Warth, B.; et al. Mycological analysis and multimycotoxins in maize from rural subsistence farmers in the former Transkei, South Africa. J. Agric. Food Chem. 2013, $61,8232-8240$.

8. Shephard, G.S.; Burger, H.M.; Gambacorta, L.; Gong, Y.Y.; Krska, R.; Rheeder, J.P.; Solfrizzo, M.; Srey, C.; Sulyok, M.; Visconti, A.; et al. Multiple mycotoxin exposure determined by urinary biomarkers in rural subsistence farmers in the former Transkei, South Africa. Food Chem. Toxicol. 2013, 62, 217-225.

9. Vend1, O.; Crws, C.; MacDonald, S.; Krska, R.; Berthiller, F. Occurrence of free and conjugated Fusarium toxins in cereal-based food. Food Add. Contam. Part A 2010, 27, 1148-1152.

10. Falavigna, C.; Cirlini, M.; Galaverna, G.; Dall'Asta, C. Masked fumonisins in processed food: Co-occurrence of hidden and bound forms and their stability under digestive conditions. World Mycotoxin J. 2012, 5, 325-334.

11. Berthiller, F.; Crews, C.; Dall'Asta, C.; De Saeger, S.; Haesaert, G.; Karlovsky, P.; Oswald, I.P.; Seefelder, W.; Speijers, G.; Stroka, J. Masked mycotoxins: A review. Mol. Nutr. Food Res. 2013, 57, 165-186.

12. Turner, P.C.; Flannery, B.; Isitt, C.; Ali, M.; Pestka, J. The role of biomarkers in evaluating human health concerns from fungal contaminants in food. Nutr. Res. Rev. 2012, 25, 162-179.

13. Polychronaki, N.; Wild, C.P.; Mykkänen, H.; Amra, H.; Abdel-Wahhab, M.; Sylla, A.; Diallo, M.; El-Nezami, H.; Turner, P.C. Urinary biomarkers of aflatoxin exposure in young children from Egypt and Guinea. Food Chem. Toxicol. 2008, 46, 519-526.

14. Duarte, S.C.; Alves, M.R.; Pena, A.; Lino, C.M. Determinants of ochratoxin A exposure-A one year follow-up study of urine levels. Int. J. Hyg. Environ. Health 2012, 215, 360-367. 
15. Riley, R.T.; Torres, O.; Showker, J.L.; Zitomer, N.C.; Matute, J.; Voss, K.A.; Gelineau-van Waes, J.; Maddox, J.R.; Gregory, S.G.; Ashley-Koch, A.E. The kinetics of urinary fumonisin B1 excretion in humans consuming maize-based diets. Mol. Nutr. Food Res. 2012, 56, 1445-1455.

16. Warth, B.; Sulyok, M.; Krska, R. LC-MS/MS-based multibiomarker approaches for the assessment of human exposure to mycotoxins. Anal. Bioanal. Chem. 2013, 405, 5687-5695.

17. Gambacorta, L.; Solfrizzo, M.; Visconti, A.; Powers, S.; Cossalter, A.M.; Pinton, P.; Oswald, I.P. Validation study on urinary biomarkers of exposure for aflatoxin B1, ochratoxin A, fumonisin B1, deoxynivalenol and zearalenone in piglet. World Mycotoxin J. 2013, 6, 299-308.

18. Solfrizzo, M.; Gambacorta, L.; Warth, B.; White, K.; Srey, C.; Sulyok, M.; Krska, R.; Gong, Y.Y. Comparison of single and multi-analyte methods based on LC-MS/MS for mycotoxin biomarker determination in human urine. World Mycotoxin J. 2013, 6, 355-366.

19. Solfrizzo, M.; Gambacorta, L.; Lattanzio, V.M.T.; Powers, S.; Visconti, A. Simultaneous LC-MS/MS determination of aflatoxin M1, ochratoxin A, deoxynivalenol, de-epoxydeoxynivalenol, $\alpha$ and $\beta$-zearalenols and fumonisin B1 in urine as a multi-biomarker method to assess exposure to mycotoxins. Anal. Bioanal. Chem. 2011, 401, 2831-2841.

20. Abia, A.W.; Warth, B.; Sulyok, M.; Krska, R.; Tchana, A.; Njobeh, P.B.; Turner, P.C.; Kouanfack, C.; Eyongetah, M.; Dutton, M.; et al. Bio-monitoring of mycotoxin exposure in Cameroon using a urinary multi-biomarker approach. Food Chem. Toxicol. 2013, 62, 927-934.

21. Ediage, E.N.; Di Mavungu, J.D.; Song, S.; Sioen, I.; De Saeger, S. Multimycotoxin analysis in urines to assess infant exposure: A case study in Cameroon. Environ. Int. 2013, 57-58, 50-59.

22. Scientific Co-operation on Question relating to Food (SCOOP, Directive 93/5/EEC). Scoop Task 3.2.10: Collection of occurrence data of Fusarium toxin in food and assessment of dietary intake by the population of EU member states. Available online: http://ec.europa.eu/food/fs/ scoop/task3210.pdf (accessed on 22 January 2014).

23. Food and Agriculture Organization; World Health Organization (FAO/WHO). Safety Evaluation of Certain Food Additives and Contaminants; Food Additives Series 59; FAO/WHO: Geneva, Switzerland, 2008.

24. Brera, C.; Angelini, S.; Debegnach, F.; De Santis, B.; Turrini, A.; Miraglia, M. Valutazione analitica dell'esposizione del consumatore alla fumonisina B1. In Proceedings of I Congresso Nazionale_Le micotossine nella filiera agro-alimentare, Superiore di Sanità, Roma, Italy, 29-30 November 2004; pp. 44-52.

25. Turner, P.C.; Rothwell, J.A.; White, K.L.M.; Gong, Y.Y.; Cade, J.E.; Wild, C.P. Urinary deoxynivalenol is correlated with cereal intake in individuals from the United Kingdom. Environ. Health Perspect 2008, 116, 21-25.

26. Turner, P.C.; Burley, V.J.; Rothwell, J.A.; White, K.L.M.; Cade, J.E.; Wild, C.P. Dietary wheat reduction decreases the level of urinary deoxynivalenol in UK adults. J. Expo. Sci. Environ. Epidemiol. 2008, 18, 392-399.

27. Turner, P.C.; White, K.L.; Burley, V.J.; Hopton, R.P.; Rajendram, A.; Fisher, J.; Cade, J.E.; Wild, C.P. A comparison of deoxynivalenol intake and urinary deoxynivalenol in UK adults. Biomarkers 2010, 15, 553-562. 
28. Turner, P.C.; Hopton, R.P.; Lecluse, Y.; White, K.L.M.; Fisher, J.; Lebailly, P. Determinants of urinary deoxynivalenol and de-epoxy deoxynivalenol in male farmers from Normandy, France. J. Agric. Food Chem. 2010, 58, 5206-5212.

29. Turner, P.C.; Gong, Y.Y.; Pourshams, A.; Jafari, E.; Routledge, M.N.; Malekzadek, R.; Wild, C.P.; Boffetta, P.; Islami, F. A pilot survey study for Fusarium mycotoxin biomarkers in women from Golestan, Northern Iran. World Mycotoxin J. 2012, 5, 195-199.

30. Wallin, S.; Hardie, L.J.; Kotova, N.; Warensjö Lemming, E.; Nälsén, C.; Ridefelt, P.; Turner, P.C.; White, K.L.M.; Olsen, M. Biomonitoring study of deoxynivalenol exposure and association with typical cereal consumption in Swedish adults. World Mycotoxin J. 2013, 6, 439-448.

31. Hepworth, S.J.; Hardie, L.J.; Fraser, L.K.; Burley, V.J.; Mijal, R.S.; Wild, C.P.; Azad, R.; McKinney, P.A.; Turner, P.C. Deoxynivalenol exposure assessment in a cohort of pregnant women from Bradford, UK. Food Addit. Contam. Part A 2012, 29, 269-276.

32. Food and Agriculture Organization; World Health Organization (FAO/WHO). Ochratoxin A. In Safety Evaluation of Certain Mycotoxins in Food; World Health Organisation: Geneva, Switzerland, 2001; pp. 281-387.

33. Gong, Y.Y.; Torres-Sanchez, L.; Lopez-Carrillo, L.; Peng, J.H.; Sutcliffe, A.E.; White, K.L.; Humpf, H.U.; Turner, P.C.; Wild, C.P. Association between tortilla consumption and human urinary fumonisin B1 levels in a Mexican population. Cancer Epidem. Biomar. 2008, 17, 688-694.

34. Van der Westhuizen, L.; Shephard, G.S.; Rheeder, J.P.; Somdyala, N.I.M.; Marasas, W.F.O. Sphingoid base levels in humans consuming fumonisin-contaminated maize in rural areas of the former Transkei, South Africa: A cross-sectional study. Food Addit. Contam. Part A 2008, 25, 1385-1391.

35. European Food Safety Authority (EFSA). Opinion of the Scientific Panel on Contaminants in the Food Chain on a request from the Commission related to Zearalenone as undesirable substance in animal feed. EFSA J. 2004, 89, 1-35.

36. Bandera, E.V.; Chandran, U.; Buckley, B.; Lin, Y.; Isukapalli, S.; Marshall, I.; King, M.; Zarbl, H. Urinary mycoestrogens, body size and breast development in New Jersey girls. Sci. Total Environ. 2011, 409, 5221-5227.

37. European Food Safety Authority (EFSA). Opinion of the Scientific Panel on contaminants in the Food Chain of the EFSA on a request from the Commission related to ochratoxin $\mathrm{A}$ in food. Available online: http://www.efsa.europa.eu/de/scdocs/doc/365.pdf (accessed on 22 January 2014).

38. Fazekas, B.; Tar, A.; Kovács, M. Ochratoxin A content of urine samples of healthy humans in Hungary. Acta. Vet. Hung. 2005, 53, 35-44.

39. Duarte, S.C.; Pena, A.; Lino, C.M. Ochratoxin A in Portugal: A review to assess human exposure. Toxins 2010, 2, 1225-1249.

40. Pena, A.; Seifrtovà, M.; Lino, C.; Silveira, I.; Solich, P. Estimation of ochratoxin A in portuguese population: New data on the occurrence in human urine by high performance liquid chromatography with fluorescence detection. Food Chem. Toxicol. 2006, 44, 1449-1454.

41. Manique, R.; Pena, A.; Lino, C.M.; Moltó, J.C.; Mañes, J. Ochratoxin A in the morning and afternoon portions of urine from Coimbra and Valencian populations. Toxicon 2008, 51, 1281-1287. 
42. Coronel, M.B.; Marin, S.; Tarragó, M.; Cano-Sancho, G.; Ramos, A.J.; Sanchis, V. Ochratoxin A and its metabolite ochratoxin alpha in urine and assessment of the exposure of inhabitants of Lleida, Spain. Food Chem. Toxicol. 2011, 49, 1436-1442.

43. Gilbert, J.; Brereton, P.; MacDonald, S. Assessment of dietary exposure to ochratoxin A in the UK using a duplicate diet approach and analysis of urine and plasma samples. Food Addit. Contam. 2001, 18, 1088-1093.

44. Domijan, A.M.; Peraica, M.; Miletić-Medved, M.; Lucić, A.; Fuchs, R. Two different clean-up procedures for liquid chromatographic determination of ochratoxin A in urine. J. Chromatogr. B 2003, 798, 317-321.

45. Hatem, N.L.; Hassab, H.M.; Abd Al-Rahman, E.M.; El-Deeb, S.A.; El-Sayed Ahmed, R.L. Prevalence of aflatoxins in blood and urine of Egyptian infants with protein-energy malnutrition. Food Nutr. Bull. 2005, 26, 49-56.

(C) 2014 by the authors; licensee MDPI, Basel, Switzerland. This article is an open access article distributed under the terms and conditions of the Creative Commons Attribution license (http://creativecommons.org/licenses/by/3.0/). 